\title{
Development of New Inspection Method for LSAW Pipes Using Matrix Phased Array UT
}

\author{
Yutaka MATSUI* and Yukinori IIZUKA \\ Steel Research Laboratory, JFE Steel Corporation, 1-1 Minamiwatarida-cho, Kawasaki-ku, Kawasaki, $210-0855$ Japan. \\ (Received on July 6, 2021; accepted on September 7, 2021)
}

\begin{abstract}
A new method was developed for inspection of welds in LSAW (Longitudinal Submerged Arc Welded) steel pipes using ultrasonic testing. Defects called I.P. (Incomplete Penetration) may occur in the central part of the wall thickness in welds of LSAW steel pipes, and these defects have a flat shape in the thickness direction. In order to detect these defects with high sensitivity without influence of the welded part shape, transmission of the ultrasonic wave perpendicular to the defect at a high refraction angle is effective. On the other hand, there were also problems in use of the angle beam method with a high refraction angle, including a decrease the sensitivity of ultrasonic testing and the need to maintain the angle accurately. In the new method proposed here, a sector scan with a line focus beam using matrix phased array UT was adopted, securing high sensitivity and stability in inspections. It's characterized by using a matrix array probe in which the small vibrators are arranged so that the width of small vibrators gradually decreases from the center of the matrix probe toward the outside. In the verification test using the actual steel pipes, good result was obtained with a $\mathrm{S} / \mathrm{N}$ ratio of $24 \mathrm{~dB}$ or more and good repeatability for a flat bottom hole with $3 \mathrm{~mm}$ diameter that is simulated I.P.. The operability of probe setting is also better than tandem method because only one probe is used and the incident angle can be adjusted by the phased array technology.
\end{abstract}

KEY WORDS: inspection; ultrasonic testing; LSAW steel pipe; phased array UT; matrix array probe.

\section{Introduction}

In this research, a new inspection method for the middle zone of the wall thickness of the weld in LSAW (Longitudinal Submerged Arc Welding) steel pipes using matrix phased array UT was developed.

Demand for LSAW steel pipes for natural gas pipelines is increasing. Because natural gas pipelines have often been constructed in severe environments in recent years, high quality is required in both the base metal and the welded seam. The inspection methods for LSAW steel pipes include ultrasonic testing for the base metal, ultrasonic testing and radiographic testing for the welded seam, ultrasonic testing for the pipe end, and magnetic particle inspection. In particular, ultrasonic testing of the welded seam plays the most important role in quality assurance.

Welding an LSAW pipe after forming the base material into the pipe shape may produce parallel plane defects on the abutting surface at the center of wall thickness. Generally, the tandem method is applied to LSAW welded seam inspection of the middle zone of wall thickness. ${ }^{1,2)}$ Two probes are used in the tandem method, and the transmission

\footnotetext{
* Corresponding author: E-mail: yu-matsui@jfe-steel.co.jp
}

and reception of the ultrasonic wave are arranged separately, as shown Fig. 1, making it possible to capture the mirrorreflected ultrasonic wave with high sensitivity. ${ }^{3)}$ However, this method has several issues in actual inspection using automatic equipment. For example, first, the defect may not be detected because the beam path of the ultrasonic wave is changed by the bead shape. ${ }^{4)}$ Second, the beam width was narrow in the circumferential direction in the past, so it is necessary to track the seam position accurately during actual inspections. Considering the problem of off seam, a wider cover range is needed in inspection of the circumferential direction. The direct incident method using a single ultra-

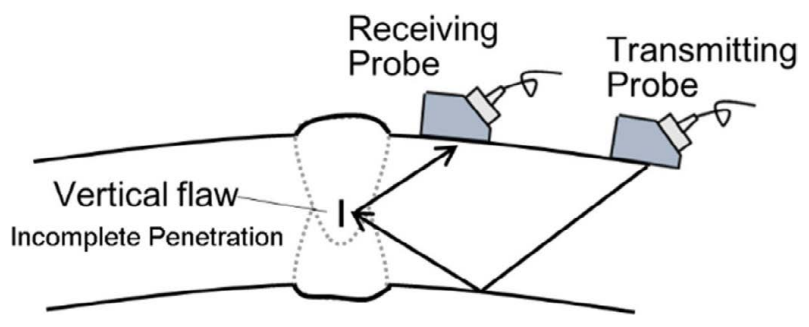

Fig. 1. Detection of vertical flaw (incomplete penetration, etc.) in middle zone of wall thickness of LSAW steel pipe by tandem method. (Online version in color.) 
sonic probe was developed to resolve these issues, and is now used in actual operation. ${ }^{5)}$ The features of this method are as described below.

In the direct incident method, the ultrasonic wave can hit a defect in middle zone of wall thickness of the weld, and the bead shape does not affect inspection detectability. The beam width for the circumferential direction is substantially wider than that in the tandem method. ${ }^{4}$ Although the direct incident method has several advantages, it has the disadvantage that the amplitude of the reflection echo decreases by $25 \%$ when the incidence angle to the pipe changes by only $\pm 0.3^{\circ}$. . $^{2}$ Moreover, considering the mechanical backlash of the automatic ultrasonic tester, it is difficult to control deviations of the incidence angle to the pipe in on-line inspection.

To solve these problems, we propose a new method which is a combination of the direct incident method and sectorial scan using matrix phased array UT. The following describes the performance of the proposed method based on laboratory tests and verification with actual equipment.

\section{Direct Incident Method for LSAW Pipe Weld Zone}

\subsection{Basic Theory of Direct Incident Method}

Figure 2 shows the direct incident method for the middle zone of the wall thickness of the weld in LSAW pipes. In this method, it is possible to transmit and receive a mirror reflection because the ultrasonic wave is transmitted perpendicularly to a planar defect at a high refraction angle.

In this figure, $D$ is the outside diameter of the pipe, $t$ is the wall thickness, $Y_{c}$ is the distance from the incidence position of a probe on the circumference, and $\theta_{r}$ is the refraction angle. $\varphi$ is the angle formed by the straight line OA and the straight line $\mathrm{OB}$. The refraction angle $\theta_{r}$ and the probe setting position $Y_{c}$ incident directly on an I.P. are calculated, and the probe is set based on the calculation result. $Y_{t}$ is the propagation distance of ultrasonic wave in the pipe. The calculations for refraction angle, the probe position and the propagation distance are described below.

In Fig. 2, when we focus on the center position of the pipe and the position of the I.P. and the probe setting position,

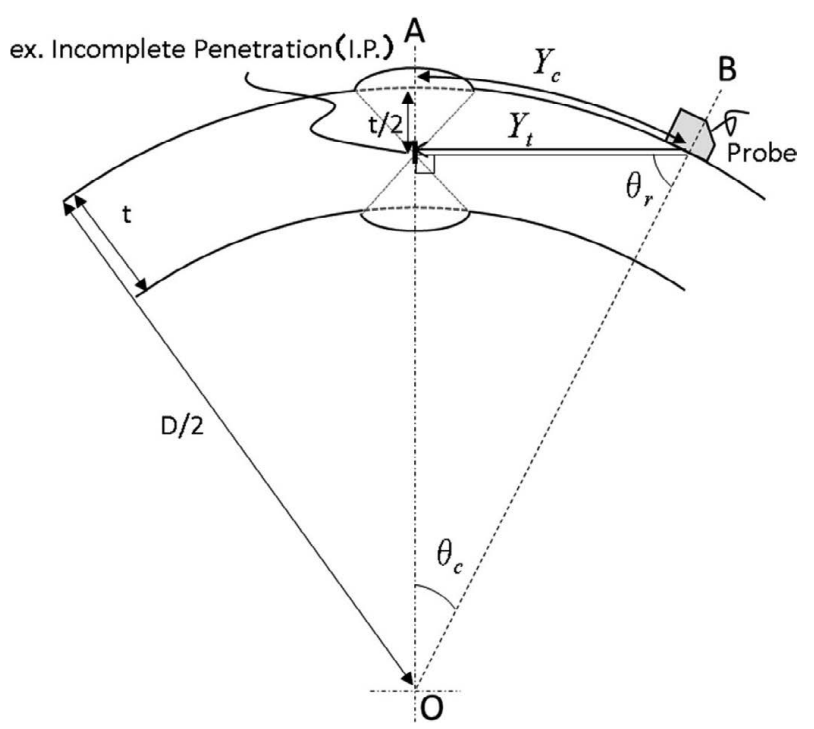

Fig. 2. Arrangement of probe position and refraction angle in direct incident method.
Eq. (1) holds. Furthermore, Eq. (1) can be modified as Eq. (2) in order to calculate the refraction angle. In this case, the refraction angle $\theta_{r}$ is calculated by Eq. (2), the probe setting position $Y_{c}$ is calculated as a ratio to the circumferential length defined by Eq. (3), and Eq. (4) is derived. $\theta_{c}$ is calculated simply based on the sum of angle of a right triangle if reflation angle is known. Therefore, Eq. (3) can be modified into Eq. (4). When probe sets in the probe setting position $Y_{c}$, the propagation distance of ultrasonic wave in the pipe is calculated by Eq. (5). When an ultrasonic wave with the calculated position and refraction angle is transmitted, the ultrasonic wave (shear wave) is incident on the steel pipe, hits the vertical defect at the center of thickness, and is reflected by the defect.

$$
\begin{gathered}
\operatorname{Sin} \theta_{r}=\left(\frac{D}{2}-\frac{t}{2}\right) / \frac{D}{2} \\
\theta_{r}=\operatorname{Sin}^{-1}\left(1-\frac{t}{D}\right)
\end{gathered}
$$

$$
\begin{gathered}
Y_{c}=\pi D \cdot \frac{\theta_{c}}{2 \pi} \ldots \\
Y_{c}=\frac{D}{2} \cdot\left(\frac{\pi}{2}-\theta_{r}\right) \\
Y_{t}=\sqrt{\frac{t}{2}\left(D-\frac{t}{2}\right)}
\end{gathered}
$$

In order to make the direct incident method easier to understand, it's going to explain concretely with the simulation results. When a pipe with a diameter of $1219.2 \mathrm{~mm}$ and a wall thickness of $18.5 \mathrm{~mm}$ is inspected by the direct incident method, the refraction angle $\theta_{r}$ is $80^{\circ}$ and the probe setting position $Y$ is located on the circumference $106 \mathrm{~mm}$ from the center of the weld. Figure 3 shows the result of a numerical calculation of the ultrasonic wave propagation under these arrangement conditions. Details of the numerical calculation conditions are shown in Table 1. Figure 3(a) shows the propagation of ultrasonic wave after $9.96 \mu$ sec after the ultrasonic wave is transmitted. A longitudinal wave is transmitted from the probe and converted into a shear wave when it is incident on the steel pipe from the wedge. The shear wave incident on the steel pipe propagates as shown in Figs. 3(b) and 3(c). At the time of Fig. 3(c), the shear wave is almost parallel to the flat bottomed hole, the wave surface of the shear wave and surface of the flat bottomed hole are directly incident on the flat bottom hole, and the reflected wave is generated.

Figure 4 shows the relationship between the refraction angle in the direct incident method and the steel pipe size. Generally, either one probe or two probes (in the tandem method) with a refraction angle of less than $70^{\circ}$ is used. In the case of large-diameter pipes with a thin wall thickness, it is necessary to use a large refraction angle in order to transmit the ultrasonic wave perpendicularly, as shown Fig. 2. According to the inspection standards of the oil majors, the middle zone of the weld must be inspected when the wall thickness is $12 \mathrm{~mm}$ or more. ${ }^{2)}$ If the wall thickness is 

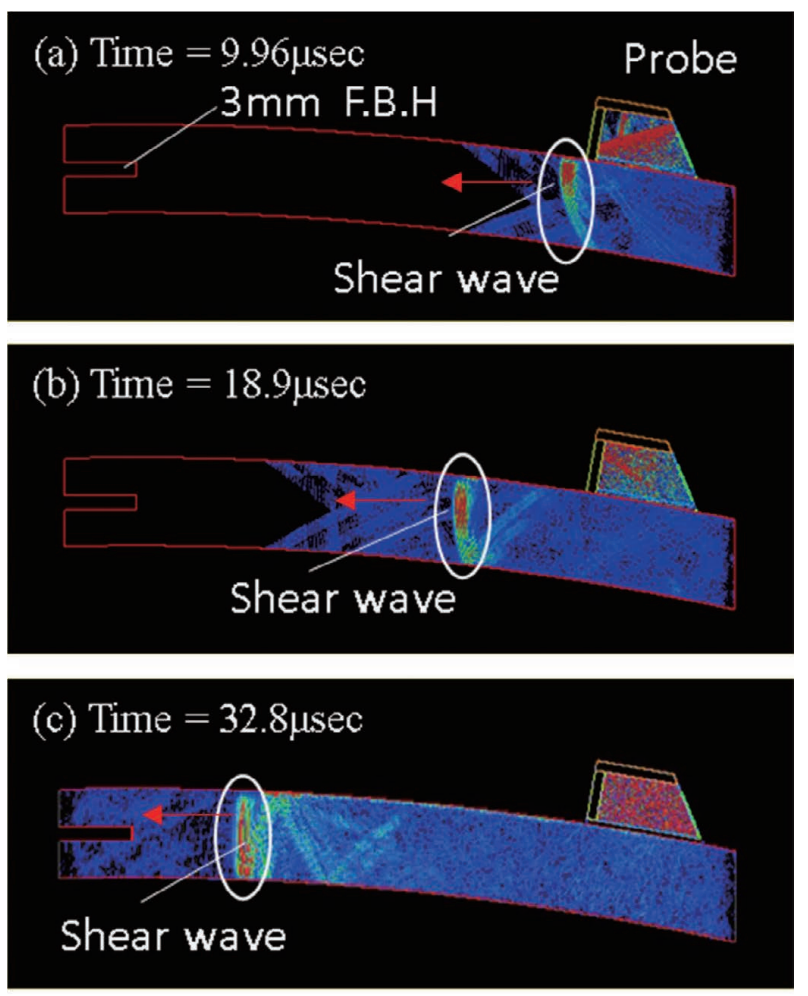

(d) Time $=40.8 \mu \mathrm{sec}$

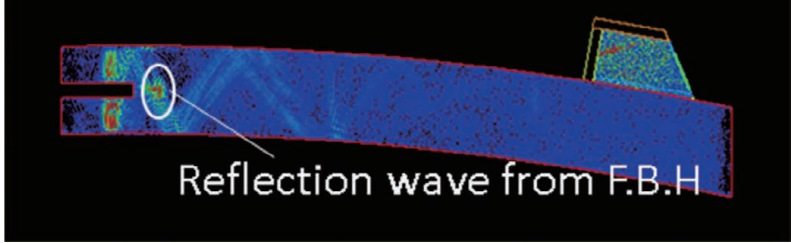

Fig. 3. Ultrasonic propagation in direct incident method calculated by numerical simulation.

Table 1. Conditions of numerical calculation of ultrasonic wave propagation.

\begin{tabular}{cc}
\hline Parameter & Value \\
\hline Frequency of ultrasonic wave & $5 \mathrm{MHz}$ \\
\hline Steel pipe size & O.D. 1 219.2 $\mathrm{mm}, \mathrm{t}=18.5 \mathrm{~mm}$ \\
\hline Sound velocity of steel & $\begin{array}{c}\text { Longitudinal wave, } 5900 \mathrm{~m} / \mathrm{sec} \\
\text { Share wave, } 3230 \mathrm{~m} / \mathrm{sec}\end{array}$ \\
\hline Sound velocity of Wedge & Longitudinal wave, $2340 \mathrm{~m} / \mathrm{sec}$ \\
& Share wave, $1150 \mathrm{~m} / \mathrm{sec}$ \\
\hline Sound velocity of water & Longitudinal wave, $1490 \mathrm{~m} / \mathrm{sec}$ \\
\hline Probe height & $8 \mathrm{~mm}$ \\
\hline
\end{tabular}

$12 \mathrm{~mm}$ or more, a maximum refraction angle of up to about 83 degrees is required to detect the incomplete penetration. Although a refraction angle larger than $70^{\circ}$ is used, it has been reported that the sensitivity are reduced. $\left.{ }^{6}\right)$ Moreover, reduced sensitivity is also a possibility in this case. Therefore, at the JFE Steel West Japan Works (Fukuyama) UOE pipe mill, digital signal processing was adopted in the AUT (automatic ultrasonic tester) to enhance sensitivity.

\subsection{Issues of Direct Incident Method}

The direct incident method has many advantages over the

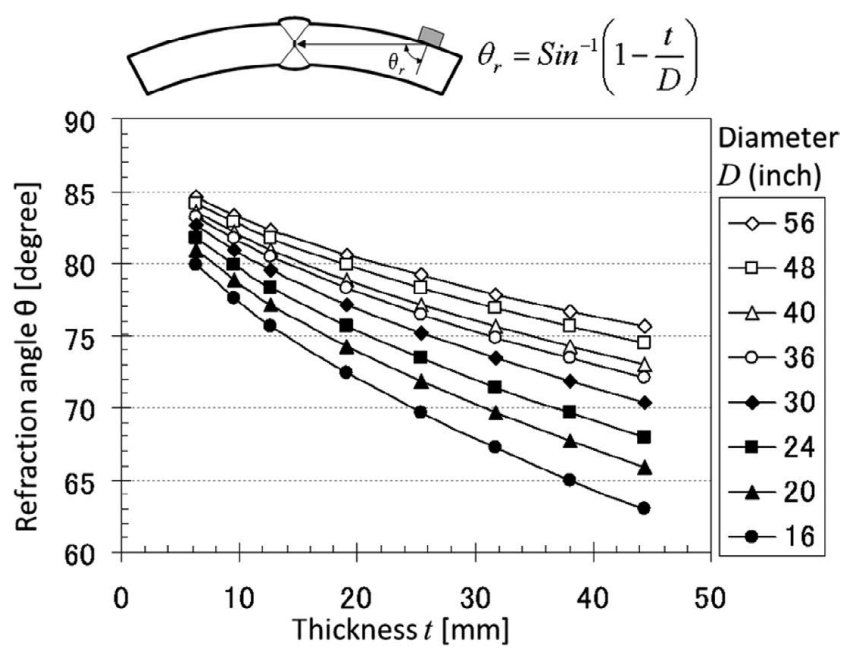

Fig. 4. Relationship between refraction angle used for flaw detection and steel pipe size (outer diameter, wall thickness) in direct incident method.

general angle beam method and the tandem method. Specifically, this method has a wide beam and can transmit an ultrasonic wave that is incident perpendicularly to a natural defect, and adjustment of the probe angle and position is easy because the ultrasonic wave is transmitted and received using only one probe. In addition, since the ultrasonic is not reflected from the inner or outer surfaces of the pipe, there is no effect of angle changes due to the bead shape.

On the other hand, the direct incident method also has several issues. First, in order to secure good repeatability, strict control of deviation of the incidence angle to the pipe is necessary in the AUT. However, in the direct incident method, the incident angle may be changed by the backlash of the seam tracking mechanism of the AUT or the pipe shape. The amplitude of the reflection angle changes sharply if the refraction angle changes. ${ }^{4}$

In addition, there is also an issue in the adjustment of the incidence angle. Because the refraction angle varies with each pipe size and wall thickness, as shown Fig. 4, it is necessary to adjust the needed incident angle for each diameter and wall thickness. When using a single probe, the acoustic contact method is the water gap method, the probe holder has an incident angle adjustment mechanism, and it is possible to adjust the refraction angle. However, this incident angle adjustment is done manually by the operator, and the sensitivity of adjustments depends on the skill of the operator. So, there is an issue that the performance of inspection depends on the skill level of the operator.

\section{Direct Incident Method Using Matrix Phased Array UT}

\subsection{Concept of New Method}

In order to solve the above-mentioned issues of the direct incident method using a single probe, we proposed the new inspection method using matrix phased array UT. Figure 5 shows the concept of the proposed method. A distinctive feature of this method is the use of a combination of sector scan in the cross section in the circumferential direction and beam focusing in the tube axial direction. Sector scan is a scanning method by swinging an ultrasonic beam within a 


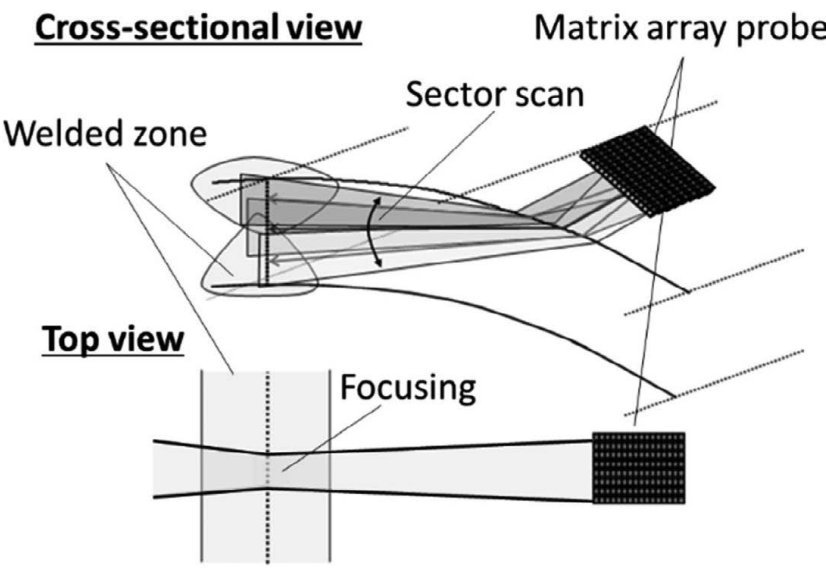

Fig. 5. Concept of new technique using combination of matrix phased array UT technique and direct incident method. Welded zone are inspected by sectorial scan with line focus beam.

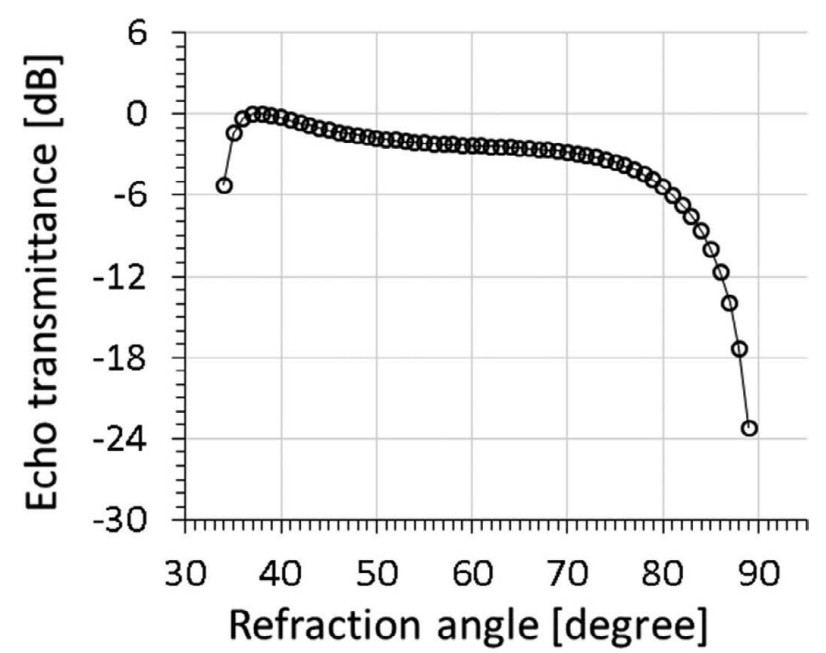

Fig. 6. Echo transmittance when ultrasonic waves are incident on steel from polystyrene wedge.

specified angle range. ${ }^{7)}$

Adoption of sector scan enables automatic adjustment of the incident angle. Furthermore, ultrasonic inspection while swinging the refraction angle can suppress fluctuations of the reflection echo amplitude by changing the incident angle and thereby realize defect detection with good repeatability.

As described in Chapter 2, the efficiency of transmission and reception (the echo transmittance) begins to gradually reduce when the refraction angle exceeds 70 degrees. And, when the refraction angle exceeds 80 degrees, the echo transmittance greatly reduces. Figure $\mathbf{6}$ shows the calculated result of the relationship between the refraction angle and echo transmittance when the ultrasonic wave is incident to the steel through the polystyrene wedge. Krautkrämer's formula of transmittance for the incident angle of the ultrasonic wave ${ }^{8)}$ was used in the calculation. In this calculation, the longitudinal wave velocity of the wedge is $2340 \mathrm{~m} /$ sec, and the shear wave velocity of the steel is $3430 \mathrm{~m} /$ sec. The refraction angle range in general use is from $45^{\circ}$ to $70^{\circ}$. On the other hand, the refraction angle of the direct incident method may be as large as $83^{\circ}$. The sensitivity with a refraction angle of 83 degrees is about $4.2 \mathrm{~dB}$ lower than the sensitivity with a refraction angle of 60 degrees. Con- sidering the effect that the diffusion attenuation ${ }^{9)}$ increases as the refraction angle increases, an attenuation of $4.2 \mathrm{~dB}$ or more may actually occur. According to this experimental investigation, ${ }^{10)}$ when the refraction angle of 83 degrees was used, the sensitivity was about $8 \mathrm{~dB}$ lower than when the refraction angle of 74 degrees was used. In the automatic ultrasonic tester, The $\mathrm{S} / \mathrm{N}$ ratio are often reduced by various disturbances such as electrical noise and changes in the angle of incidence due to the manipulator holding the sensor often. Therefore, to reduce the effect on disturbance, the beam focusing was applied to compensate for the attenuation of ultrasonic amplitude due to the decrease in echo transmittance and the increase in diffusion attenuation.

Matrix phased array UT uses a probe that has a transmission/reception surface composed of two-dimensionally arranged transducers, and the ultrasonic beam can be controlled in three dimensions by combining electronic sector scanning and focusing. ${ }^{11)}$ Since the transmission/reception direction and the focus of the ultrasonic beam can be freely controlled in three dimensions, matrix phased array UT is applied to realize cross-sectional scanning using a line focus beam. Recently, conventional automatic ultrasonic tester using single probe for steel pipe have been replaced with devices using the phased array UT. ${ }^{12}$ ) That equipment that perform the sector scans using the linear phased array probes are the mainstream. There was no method that combined a line focus beam and a sector scan with a matrix array probe to detect the defect (I.P. etc.) in the LSAW steel pipes.

\subsection{Design of Matrix Array Probe for New Inspection Method}

Next, the optimized matrix array probe to implement the concepts described in Chapter 3.1 was designed. The range of target pipe sizes is shown in Fig. 7. The outside diameter range is from 16 inches to 64 inches, and the applicable wall thickness is from $12 \mathrm{~mm}$ to $44.5 \mathrm{~mm}$. In the case of these pipe size, ultrasonic propagation $\mathrm{Yd}$ in the steel pipe is from $49 \mathrm{~mm}$ to $180 \mathrm{~mm}$, as shown Fig. 7. Therefore, in this larger range, that is, from $49 \mathrm{~mm}$ to $180 \mathrm{~mm}$, it is necessary to control the focal point of the ultrasonic beam and to have a sufficient focusing effect. The specification of the matrix array probe was designed and optimized considering the theory of the ultrasonic propagation phenomenon. Specifically, the size and arrangement of each element and the aperture size of the probe were designed in this work.

First, the aperture of the matrix array probe for focus was decided as below. The focusing effect can be estimated by Eq. (6). ${ }^{13,14)}$ The value $\mathrm{J}$ is an indicator of the focusing effect. That is, the focusing effect increases with larger values of $J$. The value is $J$ is proportional to the square of the transmitting/receiving aperture size and inversely proportional to the wavelength and focal position.

$$
J=\frac{A^{2}}{4 \lambda f_{o p}}
$$

$A$ : Aperture of transducer

$f_{o p}$ : Focal position (focus length)

$\lambda$ : Wavelength

In designing the probe, considering the attenuation of 


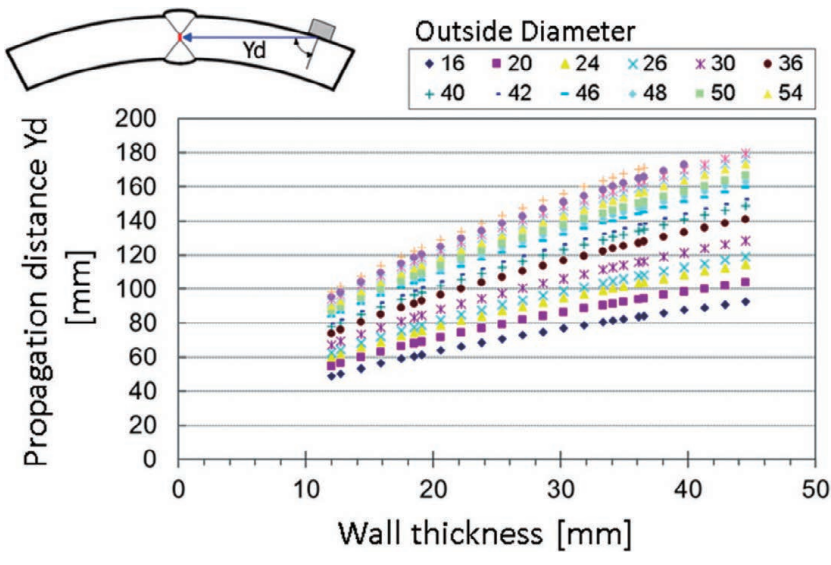

Fig. 7. Relationship of range of propagation distance of ultrasonic wave in steel pipe and size of steel pipe.

the echo transmittance and the diffusion attenuation, we thought that the compensation of at least $12 \mathrm{~dB}$ or more was necessary.

Figure 8 shows the estimated value of $\mathrm{J}$ based on the aperture size and focal position. From Fig. 8, it was understood that an array probe with an aperture of more than 30 $\mathrm{mm}$ is needed to obtain the required focusing effect of 12 $\mathrm{dB}$ more. The attenuation due to the echo transmittance can be sufficiently compensated. Next, the arrangement of the transducer elements on the aperture was designed to obtain an aperture size of about $30 \mathrm{~mm}$. In general, a probe is designed so that grating lobe does not occur. The matrix array probe for the new method has 128 elements as small transducers. These elements were arranged in an array of 16 elements $\times 8$ elements in the matrix array probe. The number of elements $8 \mathrm{Ch}$ more is sufficient for steering or focusing. ${ }^{15)}$

The side with 16 elements is used for beam focusing, and the side with 8 elements is used for the sectional scan. If the width and arrangement of the elements are determined so that the grating lobes do not occur with an aperture width of $30 \mathrm{~mm}$ and transmitting/receiving elements on the 16 Ch side, sufficient focusing is possible on the far side. But, focus control becomes difficult on the near side because the element size is large and directivity is narrow to suppress grating lobes. In this case, overlapping of the individual beams is insufficient in the near field, and the focusing effect is weak in areas that do not overlap. Conversely, if the element size is small and directivity is large, sufficient overlapping can be obtained in a larger range, but, grating lobes occur because the element size is large, directivity is narrow, and overlapping of the individual beams is insufficient in the near field. On the other hand, if the element width is reduced to widen the directivity of the individual beams, the individual beams will overlap in the near field, but grating lobe will occur. So, the element array was arranged as shown in Fig. 9 to solve these mutually-conflicting problems.

The most important feature is that the width of each element is not equal. The element width of the $\mathrm{N}$-th smallest element and its position in the array are determined so as to satisfy Eq. (7), where $\varphi_{n}$ the directivity of the N-th smallest element that has square shape. Directivity can be calculated by using the approximate expression shown in Eq. (8). $\delta_{n}$ is the an angle formed by a line connecting the center of $\mathrm{N}$-th smallest element and the focal position and the center line

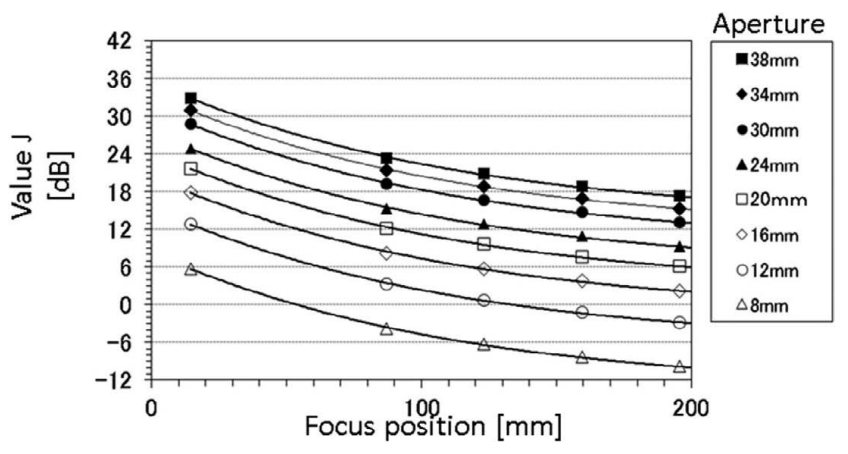

Fig. 8. Focus effect calculated from focus position and aperture size of probe.

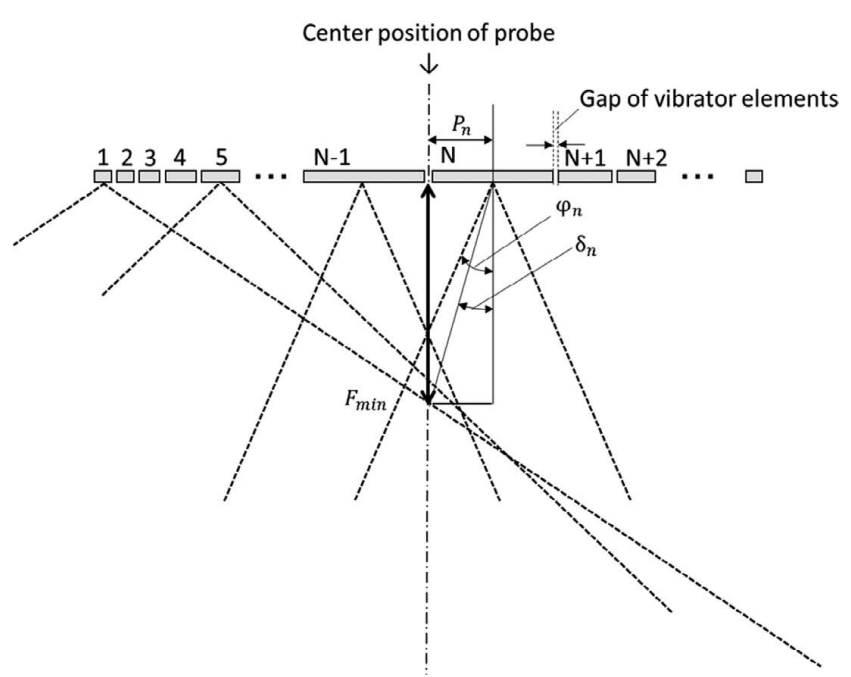

Fig. 9. Design of array element placement for wide range focus control in propagation direction, suppressing grating lobes.

of the N-th smallest element, and is described as shown in Eq. (9).

$$
\begin{array}{r}
\delta_{n} \leq \varphi_{n} \ldots \ldots . . \\
\varphi_{n}=25 \frac{\lambda}{A_{n}} \ldots . . \\
\delta_{n}=\tan ^{-1}\left(\frac{P_{n}}{F_{\text {min }}}\right)
\end{array}
$$

$\varphi_{n}$ : Directivity angle of the N-th smallest element

$\delta_{n}$ : Angle formed by a line connecting the center of $\mathrm{N}$-th small element and the focus position and the center line of the $\mathrm{N}$-th smallest element

$A_{n}$ : Aperture of the $\mathrm{N}$-th smallest element

$P_{n}$ : Distance between the center position of the $\mathrm{N}$-th transducer element and the center position of the entire aperture of the array probe

$F_{\text {min }}$ : Minimum value of focal zone

The gap between the elements is narrowed as much as possible. At distances further from the center of the probe aperture, the width of each element is narrow. The width of each element was determined so that directivity would overlap sufficiently within the range of $30 \mathrm{~mm}$ to $180 \mathrm{~mm}$ in the steel pipe. The arrangement of elements designed in this project also has an apodization effect. The method 
called apodization is used to suppress grating lobe. In the apodization, the intensity of the grating lobe is suppressed by weighting the driving voltage of each element during transmission and weighting the received waveform at each element during reception. ${ }^{16,17)}$ As the distance from the center of the aperture increases, the elements become narrower and their transmission power and receiving sensitivity decrease, providing the weighting effect used in apodization.

For the sector scan side, the arrangement of the elements was designed so that the grating lobes are as small as possible and ultrasonic waves can be transmitted and received using a polystyrene wedge in the range of refraction angles of $60-85^{\circ}$.

The designed matrix array probe was actually fabricated and examined. The next chapter discusses the contents and results of the experiment.

\section{Experiments}

\subsection{Laboratory Experiment}

In order to confirm the performance of the proposed technique, experiments were carried out with the matrix array probe and test pieces cut from pipes. In these experiments, test pieces were cut from UOE pipes, and a flat-bottomed hole with a $3 \mathrm{~mm}$ diameter was machined at the center of the wall thickness. Ultrasonic wave were transmitted and received for the test piece by the matrix array probe as shown Fig. 10. The aperture size of the matrix array probe was about $32 \mathrm{~mm}$ by $9.6 \mathrm{~mm}(16 \mathrm{Ch}$ by $8 \mathrm{Ch})$, the nominal frequency was $5 \mathrm{MHz}$, and the array elements were arranged at equal pitches on the sector scan side and at unequal pitches on the beam focus side. Of course, the width of each array element is designed to satisfy the above-mentioned Eq. (7) so that sufficient focus control can be performed in the range of the propagation distance in the steel of $30 \mathrm{~mm}$ to $180 \mathrm{~mm}$. To confirm the effect of focusing, the matrix array probe with the aperture width of $20 \mathrm{~mm}$ was also prepared. Specifically, the aperture size saw about $20 \mathrm{~mm}$ by $9.6 \mathrm{~mm}$ (16 $\mathrm{Ch}$ by $8 \mathrm{Ch}$ ), the nominal frequency was 5 $\mathrm{MHz}$, the pitch of the element arrangement is $1.2 \mathrm{~mm}$ on the focusing side, the pitch of the element arrangement is $1.2 \mathrm{~mm}$ on the steering direction. The four types of test

\section{Matrix array probe \\ Frequency $5 \mathrm{MHz}$ \\ Aperture $32 \mathrm{~mm}(16 \mathrm{CH}) \times 9.6 \mathrm{~mm}(8 \mathrm{Ch})$

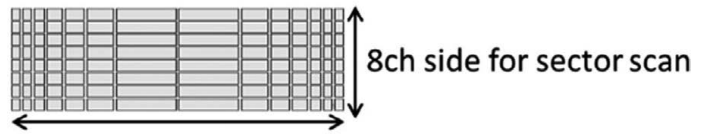 \\ $16 \mathrm{ch}$ side for focus beam}

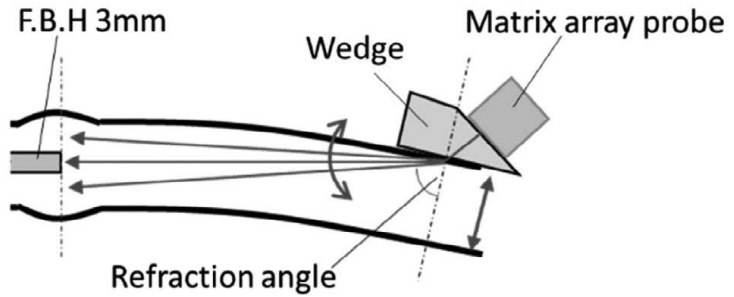

Fig. 10. Experimental method and matrix array probe specifications. pieces with the different $\mathrm{t} / \mathrm{D}$ shown Table 2 were used. As described above, when the refraction angle is $70^{\circ}$ or more, the attenuation of echo transmittance is large, the sensitivity and signal to noise $(\mathrm{S} / \mathrm{N})$ ratio will be lower. Therefore, this experiment was performed with a test piece which had a thickness and outer diameter that required ultrasonic inspection using a refraction angle of $70^{\circ}$ or more. There was a slight gap between the wedge and the contact surface of the test pieces in the experiment. Water was poured into that slight gap to realize the acoustic coupling between the wedge and the test piece.

Figure 11 shows an example of the results of the experiment which was carried out with the sector scan. This experimental result is an example under the condition of Case 1 in Table 1. Figure 11 show a scatter plot in which the angles and ultrasonic amplitudes are plotted. The ideal refraction angle calculated from Eq. (2) is $82.3^{\circ}$. However, the highest echo amplitude was obtained with the refraction angle of $81^{\circ}$. A slight difference in the shape of the pipe, a change in water temperature, or acoustic anisotropy can cause a difference in the refraction angle. In the conventional method using a single probe, the operator made fine adjustments by hand in such cases. The sector scan with the phased array probe was able to catch the appropriate refraction angle, confirming that it is possible to adjust the angle without human intervention.

Figure 12 shows an example of the A-scope image in Case 1, and Fig. 13 shows the results of a comparison of the sensitivity and the $\mathrm{S} / \mathrm{N}$ ratio in the experiments. The comparison of the sensitivity of two types of matrix array probes are shown in Fig. 13(a). The comparison of the $\mathrm{S} / \mathrm{N}$

Table 2. Pipe size and arrangement condition for direct incident method used in laboratory experiment. Arrangement condition was calculated by Eqs. (2), (4) and (5).

\begin{tabular}{ccccccc}
\hline & O.D. [mm] & W.T. $[\mathrm{mm}]$ & $\theta_{r}[$ Degree $]$ & $\mathrm{t} / \mathrm{D}[\%]$ & $Y_{c}[\mathrm{~mm}]$ & $Y_{t}[\mathrm{~mm}]$ \\
\hline Case 1 & 1219.2 & 11.1 & 82.3 & 0.9 & 82.3 & 82.1 \\
Case 2 & 1219.2 & 18.5 & 80 & 1.5 & 106.3 & 105.8 \\
Case 3 & 1422.4 & 32 & 77.8 & 2.3 & 151.1 & 150.0 \\
Case 4 & 1067 & 44.5 & 73.4 & 4.2 & 154.6 & 152.5 \\
\hline
\end{tabular}

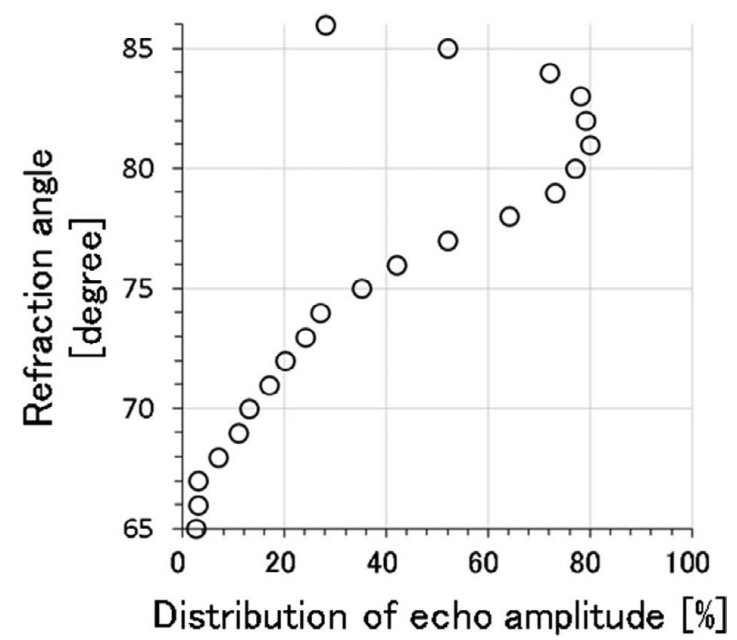

Fig. 11. B-scope image and distribution of echo amplitude for sector scan. 
ratio of two types of matrix array probes are shown in Fig. 13(b). As shown Fig. 12, the reflection echo from the flatbottomed hole with the $3 \mathrm{~mm}$ diameter was very clear and the $\mathrm{S} / \mathrm{N}$ ratio was $24.6 \mathrm{~dB}$. On the other hand, since echoes due to reverberation inside the wedge were also observed, there is room for improvement in the optimization of the reverberation absorber attached to the wedge. Next, focus on Fig. 13, the sensitivity of both probes gradually decreased as $\mathrm{t} / \mathrm{D}$ degreased. The matrix array probe with the aperture width of $32 \mathrm{~mm}$ provides a signal with a higher amplitude than the matrix array probe with the aperture width of 20 $\mathrm{mm}$. The difference in sensitivity between the two probes was $4.4 \mathrm{~dB}$ at the minimum and $7.6 \mathrm{~dB}$ at the maximum. Theoretically, it was expected to improve the sensitivity by $7 \mathrm{~dB}$. However, in the experimental results, the improvement effect was slightly small. In this proposal method, the beam is a line focus beam, so, it's considered that the focusing effect was less than that of the point focus beam. The $\mathrm{S} / \mathrm{N}$ ratio was $24 \mathrm{~dB}$ or more in all the cases tested as shown 13 (b) when using the matrix array probe with the aperture width of $32 \mathrm{~mm}$. The $\mathrm{S} / \mathrm{N}$ ratio when using a matrix probe with a small aperture $(20 \mathrm{~mm})$ was $18 \mathrm{~dB}$ or more and 24 $\mathrm{dB}$ or less. As with the sensitivity, it was confirmed that the $\mathrm{S} / \mathrm{N}$ ratio is better when the aperture is larger.

\subsection{Verification Test Using Actual Pipes}

Next, a verification test was conducted using actual pipes. This section describes the experimental method and test results. The matrix phased array probe was temporarily installed on the manipulator of an existing automatic ultrasonic tester for UOE pipes. The same probe as the matrix array probe used in the experiment with the test pieces was also used in this verification test. The acoustic coupling method was the same as that described in chapter 4.1.

Five types of pipes were prepared for the verification test. The dimensions of the five pipes were selected so as to cover the $\mathrm{t} / \mathrm{D}$ range of $0.9 \%$ to $6.9 \%$, as shown Table 3 .

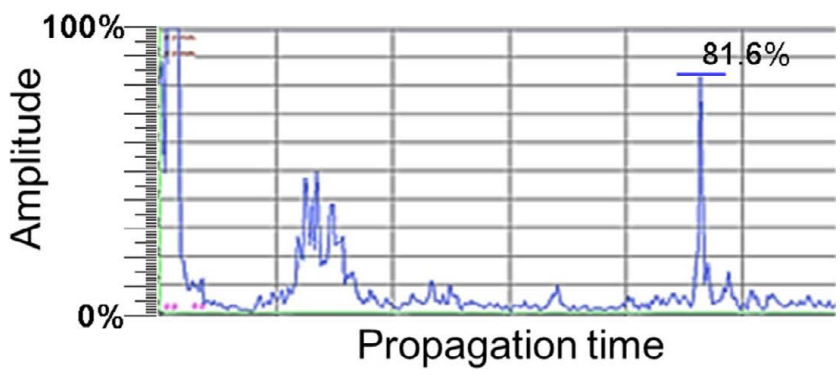

(a) Refraction echo from flat-bottomed hole (3 $\mathrm{mm}$ diameter).

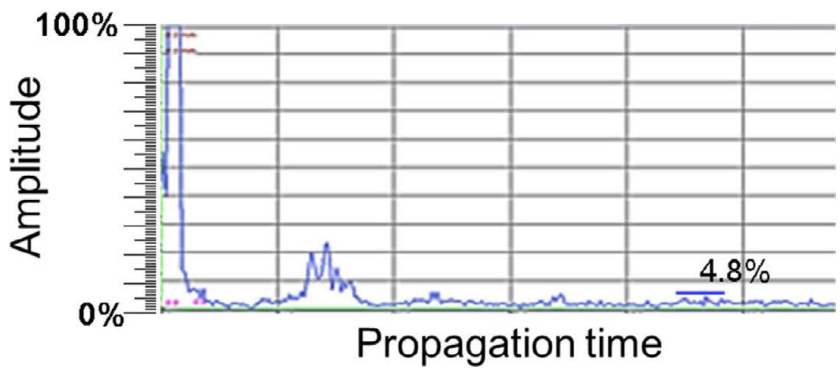

(b) Echo at part without flat-bottomed hole (3 $\mathrm{mm}$ diameter).

Fig. 12. Example of A-scope image in Case 2 (O.D. $1219.2 \mathrm{~mm}$, W.T. $11.1 \mathrm{~mm})$. (Online version in color.)
According to the standard, it is applied when the thickness is $12 \mathrm{~mm}$ or more, but in order to have $\mathrm{t} / \mathrm{D}$ variation, the steel pipes that has thinner than $12 \mathrm{~mm}$ wall thickness were also prepared. Each pipe had a flat-bottomed hole with a 3 $\mathrm{mm}$ diameter in the center of wall thickness. In this verification test, first, the calibration was carried out. Specifically, the probe arrangement, the center angle of the sector scan, the focus position, and the sensitivity were adjusted in the calibration. The sector scan pattern used three swing refraction angles of $+2^{\circ}, 0^{\circ}$, and $-2^{\circ}$, with the highest amplitude as the center refraction angle. After the calibration was completed, the inspection was conducted eight times dynamically for one pipe using the prepared test pipes. The scanning speed (the speed at which the pipe is transported) was from $10 \mathrm{~m} / \mathrm{min}$ to $12 \mathrm{~m} / \mathrm{min}$. This condition is the same as the scanning speed in actual inspections. Since the beam width of the line focus beam is narrow, the PRF and SRF were set so that the beam width overlap sufficiently for the long axis direction of the steel pipe.

Figure 14 shows the result of plotting the $\mathrm{S} / \mathrm{N}$ ratio for

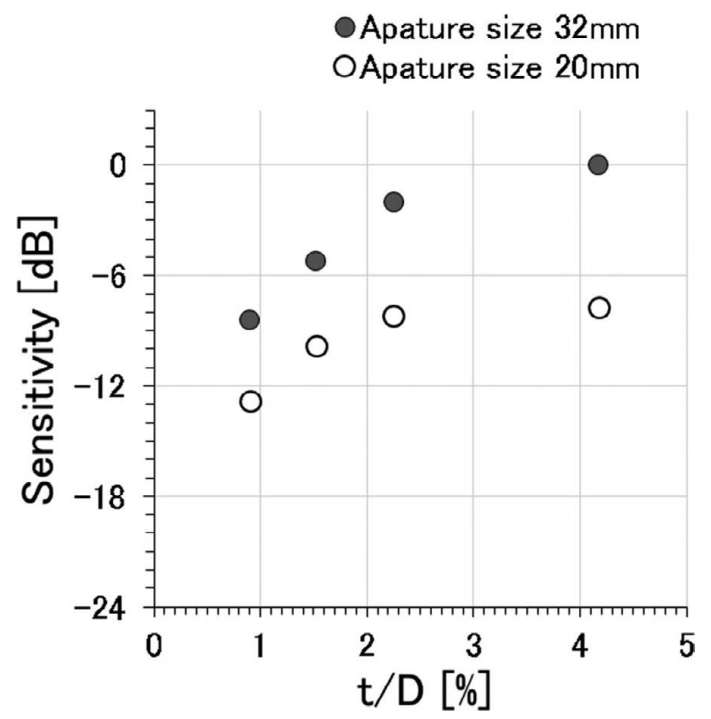

(a) Comparison of sensitivity (gain) to echo height of a flat bottom hole with a diameter of $3 \mathrm{~mm}$.

Apature size $32 \mathrm{~mm}$ OApature size $20 \mathrm{~mm}$

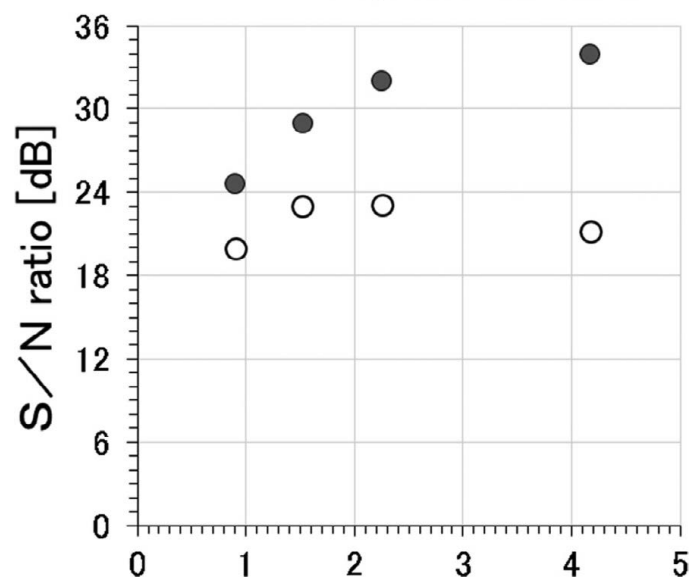

(b) Comparison of $\mathrm{S} / \mathrm{N}$ ratio for flat bottomed hole a diameter of $3 \mathrm{~mm}$.

Fig. 13. Scatter plot of Signal to Noise ratio and t/D in experiment using coupon with welds cut out from steel pipe. 
Table 3. Pipe size and arrangement condition for direct incident method used in experiment on actual product line. Arrangement condition was calculated by Eqs. (2), (4) and (5).

\begin{tabular}{lrccccc}
\hline & O.D. $[\mathrm{mm}]$ & W.T. $[\mathrm{mm}]$ & $\theta_{r}[$ Degree $]$ & $\mathrm{t} / \mathrm{D}[\%]$ & $Y_{c}[\mathrm{~mm}]$ & $Y_{t}[\mathrm{~mm}]$ \\
\hline Case 1 & 1422.4 & 12.7 & 82.3 & 0.9 & 95.1 & 94.8 \\
Case 2 & 457.2 & 6.4 & 80.4 & 1.4 & 38.3 & 38.1 \\
Case 3 & 457.2 & 11.9 & 76.9 & 2.6 & 52.3 & 51.8 \\
Case 4 & 1219.2 & 44.8 & 74.4 & 3.7 & 165.8 & 163.7 \\
Case 5 & 457.2 & 31.8 & 68.5 & 6.9 & 85.8 & 83.8 \\
\hline
\end{tabular}

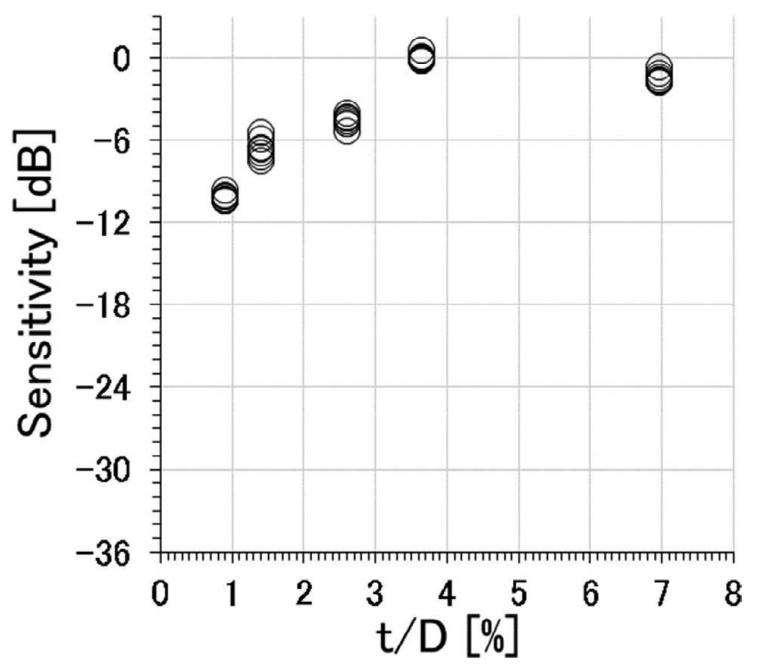

(a) Sensitivity for flat bottomed hole with a diameter of $3 \mathrm{~mm}$.

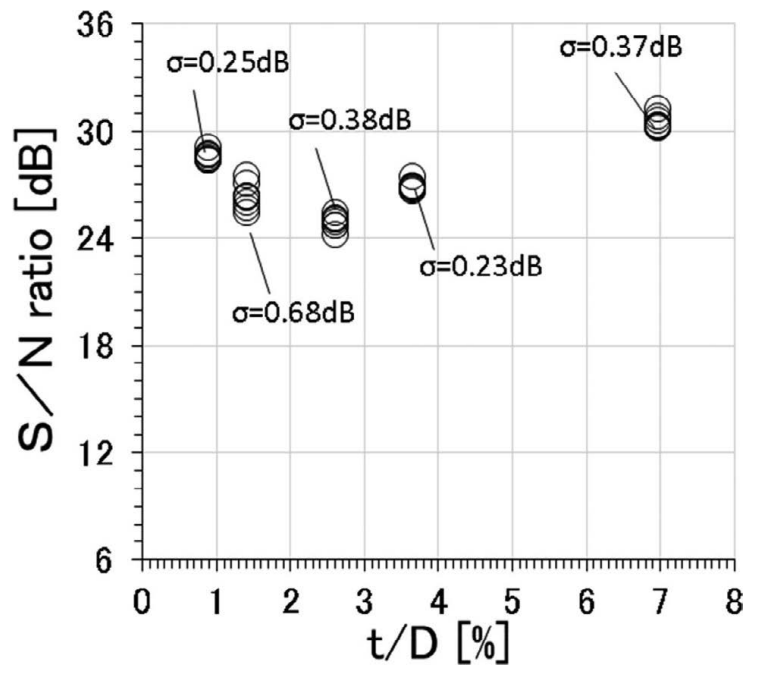

(b) $\mathrm{S} / \mathrm{N}$ ratio for flat bottomed hole with a diameter of $3 \mathrm{~mm}$.

Fig. 14. Results of new method in actual product line.

the $t / D$ of the pipes used in the experiment. Figure 14(a) shows the sensitivity, Fig. 14(b) shows the $\mathrm{S} / \mathrm{N}$ ratio. In the verification test, similar to the lab experiment results, the sensitivity gradually decreased as t/D degreased. With the proposed method, the $\mathrm{S} / \mathrm{N}$ ration was $24 \mathrm{~dB}$ or more as shown Fig. 14(b). The standard deviation of repeatability was $0.68 \mathrm{~dB}$ or less. In the laboratory experiment in Chapter 4.1 , the $\mathrm{S} / \mathrm{N}$ ratio also tended to decrease as $\mathrm{t} / \mathrm{D}$ decreased. However, No such tendency was observed in the verification test. Since the sensitivity tends to be similar to that in the laboratory experiment, it is inferred that such a result was obtained due to noise. Noise is caused by electrical noise, reverberation inside wedges, and the structure particle of steel pipes. In Case 2 and Case 3, because the propagation distance of the steel pipe is short, there are possibility that it may have been affected by the reverberation of the wedge.

As a result of the verification test, it was confirmed that the flat bottom hole can be detected with good $\mathrm{S} / \mathrm{N}$ and good repeatability in the $\mathrm{t} / \mathrm{D}$ range of $0.9 \%$ to $6.9 \%$.

\section{Conclusion}

A new method for inspection of the middle zone in the wall thickness of welds of LSAW pipes was proposed. The direct incident method using a conventional single probe has an issue of inspection stability. The new method uses a combination of matrix phased array UT and the direct incidence method to improve inspection stability. To realize this method, a matrix array probe with elements arranged at unequal pitches was designed in consideration of the focusing effect and conditions for suppressing the generation of grating lobes. In the laboratory experiments (static experiments), the result is obtained that the actual increase in sensitivity was less than the theoretically estimated value. However, using the designed matrix array probe, it was confirmed that the $\mathrm{S} / \mathrm{N}$ ratio is $24 \mathrm{~dB}$ or more for a flat bottomed hole with $3 \mathrm{~mm}$ diameter. The $\mathrm{S} / \mathrm{N}$ ratio of $24 \mathrm{~dB}$ or more and good reproducibility were obtained even for a flat bottom hole with a diameter of $3 \mathrm{~mm}$ simulated as a vertical defect (I.P.) in the verification test (dynamic experiments) using an actual steel pipe, too.

\section{REFERENCES}

1) W. A. K. Deutsch, P. Schulte, M. Joswig and R. Kattwinkel: 9th European Conf. on NDT (ECNDT 2006), (Berlin), DGZfP (German Society for Non-Destructive Testing), Berlin, (2006), Tu.2.3.1.

2) Shell DEP 31.40.20.37-GEN: 2013, LINE PIPE FOR CRITICAL SERVICE.

3) R. Raillon, M. Lozev, R. Spencer, E. Kerbrat and S. Mahaut: AIP Conf. Proc., 760 (2005), 914.

4) Y. Matsui and Y. Iizuka: Proc. 2016 11th Int. Pipeline Conf. (IPC 2016), (Calgary), The American Society of Mechanical Engineers, New York, (2016), 64328.

5) T. Hashimoto, Y. Iizuka, Y. Matsufuji, K. Maeda and S. Iwakura: CAMP-ISIJ, 13 (2000), 1041 (in Japanese).

6) K. Kimura, K. Oka and H. Hayashi: J. JSNDI, 53 (2004), 763 (in Japanese).

7) O. Von Ramm and S. W. Smith: IEEE Trans. Biomed. Eng., BME-30 (1983), No. 8, 438.

8) J. Krautkrämer and H. Krautkrämer: Ultrasonic Testing of Materials, 2nd ed., Springer-Verlag, Berlin, Heidelberg, (1977), 605.

9) I. N. Ermolov and V. G. Shcherbinskii: Sov. J. Nondestruct. Test., 6 (1971), No. 6, 665

10) M. Nagase, Y. Hirose, T. Horiyuki and M. Yamano: 18th Int. Offshore and Polar Engineering, Conf., (ISOPE 2008), (Vancouver), ISOPE, Mountain View, (2008), TPC-472.

11) R/D Tech Inc.: Introduction to Phased Array Ultrasonic Technology Applications, 1st ed., R/D Tech, Québec, (2004), 101.

12) W. A. K. Deutsch and F. Bartholomai: Fall Conf. and Quality Testing Show 2012, (Orland), ASNT, Columbus, OH, (2012), 208.

13) K. Kimura, H. Fukuhara and S. Matsumoto: J. JSNDI, 31 (1982), 2 (in Japanese).

14) J. Krautkrämer and H. Krautkrämer: Ultrasonic Testing of Materials, 2nd ed., Springer-Verlag, Berlin, Heidelberg, (1977), 75.

15) Y. Iizuka: Proc. 16th Symp. on Ultrasonic Testing, (Tokyo), JSNDI, Tokyo, (2009), 29 (in Japanese).

16) K. D. Olbrish and K. K. Shung: Proc. SPIE, 3037 (1997), 196.

17) O. Keitmann-Curdes, B. Brendel, C. Marg and H. Ermert: IEEE Ultrasonic Symp., IEEE, Piscataway, NJ, (2002), 1677. 